\title{
Review \\ The spliceosome as target for anticancer treatment
}

\author{
RJ van Alphen', EAC Wiemer', H Burger' and FALM Eskens*, \\ 'Department of Medical Oncology, Erasmus University Medical Centre, PO Box 2040, Rotterdam 3000 CA, the Netherlands
}

\begin{abstract}
The spliceosome is a ribonucleoprotein complex involved in RNA splicing, that is, the removal of non-coding introns from precursor messenger RNA. (Alternative) Splicing events may play an essential role in tumourigenesis. The recent discovery that the spliceosome is a target for novel compounds with anticancer activity opens up new therapeutic avenues. British Journal of Cancer (2009) 100, 228-232. doi:I0.1038/sj.bjc.660480 I www.bjcancer.com Published online 25 November 2008

(c) 2009 Cancer Research UK
\end{abstract}

Keywords: spliceosome; alternative splicing; planiedolide; E-7107; spliceostatin A

Most protein-coding genes in our genome are composed of multiple exons interrupted by introns. The exons are usually relatively short about $50-250$ base pairs, whereas introns are much larger and can be up to several thousands of base pairs. Together the exon sequences encode the protein, whereas the introns are generally non-coding. When a gene is transcribed, exons and intron sequences are converted into a single precursor messenger RNA (pre-mRNA). In a process called splicing, the intron sequences are removed from the pre-mRNA and the exons fused together resulting in the formation of the mature messenger RNA (mRNA), which is subsequently capped at its $5^{\prime}$ end and polyadenylated at its $3^{\prime}$ end, and transported out of the nucleus to be translated into protein in the cytoplasm. In general, most genes give rise to multiple spliced transcripts by alternative splicing. These transcripts contain different combinations of exons, and sometimes introns or part thereof, leading to different mRNA variants and the synthesis of alternative proteins (Figure 1). The mere existence of alternatively spliced products greatly increases cellular and organismal complexity and may have allowed for evolution by producing additional regulation and diversification of gene function. It is obvious that splicing and alternative splicing must be tightly regulated and executed in time and space not to interfere with the normal cellular and organismal physiology. The spliceosome, an intracellular complex of multiple proteins and ribonucleoproteins, is the main cellular machinery guiding splicing. Recently, two natural compounds interfering with the spliceosome were found to display antitumour activity in vitro and in vivo. Therefore, it is conceivable that inhibiting the spliceosome could serve as a novel target for anticancer drug development (Kaida et al, 2007; Kotake et al, 2007).

\section{SPLICING AND ALTERNATIVE SPLICING: THE ROLE OF THE SPLICEOSOME}

To direct correct processing of pre-mRNA, the intron sequences contain a number of core splicing signals, notably the conserved splice-site sequences at their extreme $5^{\prime}$ and $3^{\prime}$ ends and a conserved branch point region. The branch point sequence and the

*Correspondence: Dr FALM Eskens; E-mail: f.eskens@erasmusmc.nl Received 4 August 2008; revised 30 October 2008; accepted 4 November 2008; published online 25 November 2008 branch point itself, usually an adenosine, are located about 20-40 nucleotides upstream of the $3^{\prime}$ splice site. The critical process of recognising splice sites and the removal of introns and/or exons is the task of the spliceosome (Staley and Guthrie, 1998; Will and Lührmann, 2001). The spliceosome consists of five non-coding uridine-rich small nuclear ribonucleoproteins (U snRNPs) and a multitude of associated proteins, creating a network of RNARNA, RNA - protein and protein-protein interactions (Zhou et al, 2002; Jurica and Moore, 2003) (Figure 2). In fact, well over 200 different splicing factors interacting with the spliceosome have been identified, and it is clear that much more research is needed before we fully understand all the intricacies of RNA splicing (Jurica and Moore, 2003; Wang and Burge, 2008). The various spliceosome components, in particular, the small nuclear RNPs, are sequentially recruited to the splice sites. Next, they are assembled into the spliceosome after which splicing is initiated by a series of two transesterification reactions producing two ligated exons and a liberated intron (Staley and Guthrie, 1998; Wang and Burge, 2008) (Figure 2). Splicing at different locations in the same pre-mRNA transcript, alternative splicing, enables a gene to produce variant mature mRNAs, and consequently functionally different proteins (House and Lynch, 2008). The precise mechanisms of and/or triggers for alternative splicing are yet unknown. In higher eukaryotes, like humans, the accuracy of splicing is not solely dictated by base-pairing interactions of the U snRNPs with the pre-mRNA. Owing to degeneracy and poor definition of small splice sites at the end of long introns, splice-site recognition is also influenced by (i) the coupling of splicing with other processes such as transcription, (ii) the velocity of the splicing reaction, (iii) external stimuli, like the presence of growth factors or oxidative stress and (iv) the three-dimensional structure of pre-mRNA itself (Tazi et al, 2005b; Disher and Skandalis, 2007; House and Kristen, 2008). In the latter case, the three-dimensional folding of premRNA determines whether a (in pre-mRNA embedded) splice regulator will be located in the vicinity of a splice site.

Four regulatory sequence elements have been identified; two stimulative ones, splicing enhancers located in introns (ISE) or exons (ESE), and two suppressive ones, splicing silencers located in introns (ISS) or exons (ESS), respectively (Tazi et al, 2005b; House and Kristen, 2008 and references therein). The enhancers generally recruit spliceosome components or splicing factors to a splice site on pre-mRNA. On the other hand, the splicing silencers block specific splicing sites; hence interfere with the binding of 


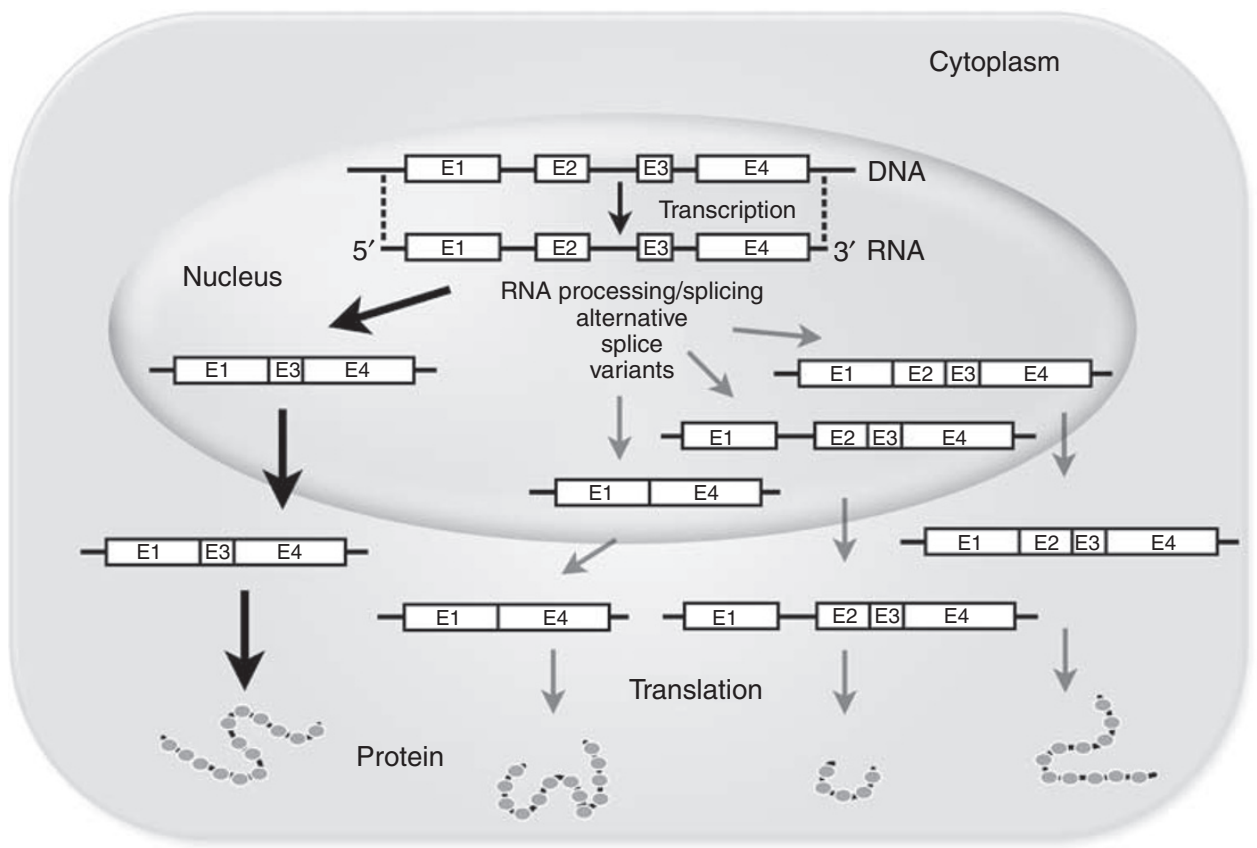

Figure I Schematic illustration of transcription, pre-mRNA processing/splicing and translation. A protein-coding gene is transcribed by RNA polymerase II into pre-mRNA. Subsequently, or already during transcription, non-coding introns are removed in a process called splicing, after which the mature mRNA is capped and polyadenylated and transported to the cytoplasm where it is translated into protein. Depicted is a gene consisting of four exons interspersed by three introns. Under normal splicing conditions, exons I, 3 and 4 are linked together forming the vast majority of mature transcripts (bold black arrow). Alternative splicing may recombine gene elements into new configurations that may or may not display the activity or characteristics of the original protein. For example, alternative splicing reactions (grey arrows) can result in the acquirement of an extra exon (exon 2), in the skipping of exons (exon 2 and 3 ) or in an extra exon and part of the first intron through the use of a cryptic splice site in the first intron. In the latter case, a truncated protein may form due to the presence of a termination codon in the remaining intron sequence. Under normal circumstances, nonsense-mediated decay (NMD) is responsible for the degradation of mRNAs that have a premature stop codon to prevent the synthesis of detrimental truncated proteins. Note that the NMD is dysfunctional in cells treated with spliceostatin A leading to the expression of truncated proteins (see text for details).

components of the spliceosome with the blocked splice site on pre-mRNA. These specific exonic and intronic pre-mRNA sequences are modulated by interactions with non-spliceosomal nuclear RNA-binding proteins. Both repressing (the heterogenous nuclear ribonucleoprotein (hnRNP) family) and stimulating (the serine/arginine (SR) protein family) proteins for these splice modulators have been identified, both regulating alternative splicing indirectly (Tazi et al, 2005b; House and Kristen, 2008).

In conclusion, the removal of introns and/or exons has to be precise and complete, otherwise incorrectly or differently joined exons yield no mature and functional mRNA, or result in altered genetic messages with the potential of initiating malignant behaviour in previous normal cells.

\section{ALTERNATIVE SPLICING AND DISEASE/CANCER}

Recent research has revealed that alternatively spliced products can be linked to various (genetic) diseases and also may play a role in cancer development (Wang and Burge, 2008). A systematic approach using large-scale sequencing and splicing-sensitive microarrays gave for the first time a view of the vast spectrum of alternative transcripts (Blencowe, 2006; Ben-Dov et al, 2008). One or more alternatively spliced exons can be found in transcripts of two-thirds of human genes. In general, the function of the encoded protein remains unchanged. However, some of the alternative protein products may display a malignant phenotype (Blencowe, 2006). Whether alternative splicing is the cause or result of malignant behaviour in cancer cells remains unclear. However, as alternative splicing affects most genes, it is likely that cell cycle control, signal transduction, angiogenesis, motility and invasion and the metastasis and apoptosis processes that are often impaired in cancer will be affected (Skotheim and Nees, 2007).
Extensive research indicates that several familial cancer syndromes are caused by somatic (point) mutations or singlenucleotide-polymorphisms (SNPs) occurring in splice sites. These changed splice-site signals may give rise to alternative splice variants of tumour suppressor genes such as $B R C A 1, A P C, p 53$, FHIT and LKB1 and the prostate cancer susceptibility gene, KLF6 (see for a recent review Skotheim and Nees, 2007). Moreover, it has been shown that splice variants of H-RAS, the enzyme phosphatase PPR2C $\gamma$, CD44v3 (a cell surface glycoprotein), SPN1 $4 \mathrm{~N}$ (a deletion mutant of snRNP-specific nuclear import adapter snurportin 1), RTVP-1 (in malignant gliomas) and CD44v6 (in various epithelial cancers) all favour tumourous behaviour in cells (Allemand et al, 2007; Barbier et al, 2007; Rino et al, 2007; Vela et al, 2007). The increasing number of known non-familial cancers that express alternatively spliced proteins, each of which may contribute to the malignant phenotype, provides additional impetus to search for agents that interfere with alternative splicing and may display an anticancer potential (Barbier et al, 2007).

Currently, three different approaches to target (alternative) splicing in cancer are explored. First, one may be able to modulate alternative splicing by identifying specific splice sites and directly or indirectly block their use. Alternatively, one may identify and characterise splice variant products (ie, proteins) that contribute to the malignant phenotype and determine how they differ from their normal protein counterparts. Structural differences may then be used to develop antibodies or small molecules that specifically target these proteins. A second approach may be the manipulation of splice modulators, for example, hnRNPs or SR proteins, hereby indirectly influencing splicing activity. A third approach is based on the simple notion that malignant cells in general have higher metabolic rates than normal cells, and consequently have increased splicing rates and are more prone to splice inhibitors or 


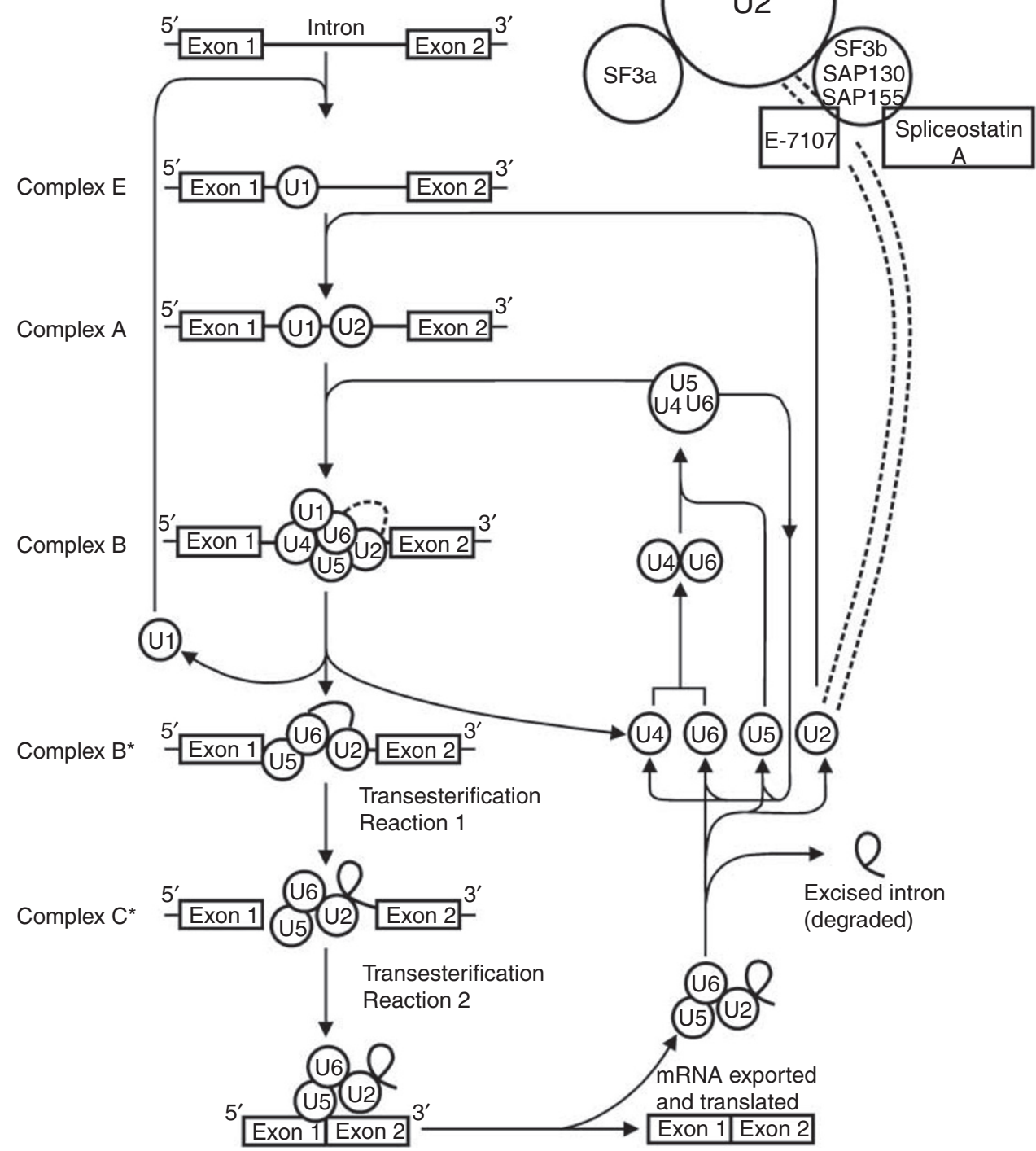

Figure 2 The spliceosome assembly cycle. Through interactions with various proteins that recognise specific splice site features, the spliceosome components, that is, small nuclear ribonucleoproteins (snRNPs) designated with UI, U2, U4, U5 and U6 are sequentially recruited to the splice site and assembled into the spliceosome. Once completed, splicing is catalysed in two consecutive transesterification reactions. In the initial step, the $2^{\prime} \mathrm{OH}$ group of the branch point adenosine upstream of the $3^{\prime}$ end of the intron reacts with the $5^{\prime}$ splice junction, forming a novel $2^{\prime}, 5^{\prime}$ phosphodiester bond between the branch point and the $5^{\prime}$ terminal nucleotide of the intron, giving rise to a lariat structure. In the second reaction, the $3^{\prime} \mathrm{OH}$ of the $5^{\prime}$ exon attacks the $3^{\prime}$ splice junction producing linked $5^{\prime}$ and $3^{\prime}$ exons and liberating the intron. Subsequently, the snRNPs involved are released and recycled in the splicing process. In the top right-hand corner, a detailed view of U2 snRNP with subcomplexes, SF3a and SF3b, is shown. The spliceosome-associated proteins (SAP) I30 and/or 155 are targeted by pladienolide derivatives (a.o. E-7/07) and spliceostatin A.

modulators. Targeting the spliceosome directly might therefore cause tumour inhibition at drug levels that do not affect normal cells.

The three strategies such as (i) splice-site modulation and targeting of variant proteins; (ii) targeting of splice modulators and (iii) spliceosome inhibitors are reviewed in detail below.

\section{SPLICE-SITE MODULATION AND TARGETING OF VARIANT PROTEINS}

The delineation of splice sites used in alternative splicing or the functional characterisation of aberrant proteins expressed as a result of alternative splicing may provide the necessary insight for therapeutic interference. Specific splice-site mutations or proteins might (i) prove to be of predictive value and (ii) become potential prognostic markers (Yu et al, 2007), (iii) prove to be targets of future (anticancer) antibody-guided drugs (Orban and Olah, 2003; Heider et al, 2004; Xiang et al, 2007) or (iv) become a target for peptide receptor radionucleotide therapy. An example of the latter is a splice variant of the cholecystokinin receptor found in colorectal and pancreatic cancer that can be targeted by radionuclide therapy (Laverman et al, 2008). A disease in which an understanding of the alternative splicing process is used for therapeutic purposes is Duchenne's muscular dystrophy (DMD). Duchenne's muscular dystrophy is a neuromuscular disease caused by deletions/duplications or point mutations in the $2.4 \mathrm{Mb} D M D$ gene, encoding dystrophin, causing disruption of 
the open reading frame (ORF). The induction of exon skipping, circumventing the mutated exon through intramuscular injection of carefully designed antisense oligonucleotides, corrects the ORF of dystrophin in in vitro cell lines, animal models and humans (van Deutekom et al, 2007). Another example concerns spinal muscular atrophy (SMA) caused by the deletion of SMA1 gene and the inability of the remaining SMA2 gene, which is virtually identical to SMA1, to compensate for the SMA1 loss, as its transcript lacks exon 7. Here, antisense oligonucleotides targeting an intronic splicing silencer (ISS-N1) may fully restore SMN2 exon 7 inclusion (Singh, 2007).

If we indeed are able to create therapeutic approaches that correct the deleterious effects of splice variants by remodelling the (alternative) splice reactions in non-malignant diseases, it is conceivable that this approach might also be applicable in malignancies; Here, splice-site modulation would be a valuable concept for intervening in the expression of various oncogene and tumour suppressor gene mutations.

\section{TARGETING OF SPLICE MODULATORS}

The SR proteins comprise a family of splice factors that modulate splice-site selection by mediating the interaction between premRNA transcripts and the spliceosome. Phosphorylation of the serines in their arginine/serine (RS) domains regulates the intracellular localisations of SR proteins and their interaction with related domains of other splice factors or RNA. This consequently affects alternative splicing of pre-mRNAs. There are several kinases, for example, Clk/Sty, SRPK1, SRPK2 and topoisomerase I that are known to phosphorylate SR proteins. Several compounds have been reported that target these kinases, for example, diospyrin (Tazi et al, 2005a), indole derivatives (Soret et al, 2005) and indolocarbazole (NB506; Pilch et al, 2001), inhibit the kinase activity of topoisomerase I. Similarly, a benzothiazole compound displayed clear inhibitory effects on the activity of Clk1/Sty (Muraki et al, 2004). It was shown that exposure to these compounds modulates the splicing profile of several genes, and alters gene expression patterns, due to inhibition of SR protein phosphorylation. In future, drugs like these or derivatives thereof may be used for the therapeutic manipulation of alternative splicing.

\section{SPLICEOSOME INHIBITORS}

Recently, two chemically different microbial natural products with profound cancer cell inhibiting potential were found to target the spliceosome. Both compounds, the pladienolide derivatives (Kotake et al, 2007) and spliceostatin A (Kaida et al, 2007), appear to bind to SF3b, a subcomplex of U2 snRNP, which is an essential component of the spliceosome (Figure 2). SF3b together with SF3a enable U2 snRNP to associate with the branch point region near the $3^{\prime}$ end of the intron. The SF3b subcomplex consists of seven proteins such as the spliceosome-associated protein (SAP) 10, SAP14a, SAP14b, SAP49, SAP130, SAP145 and SAP155. The binding of pladienolide and spliceostatin A to SF3b causes an inhibition of the spliceosomal functions resulting in impaired splicing, and thus altered gene expression patterns.

Pladienolide compounds, in particular pladienolide B and D, displayed anticancer activity (Mizui et al, 2004; Kotake et al, 2007). E-7107 is a synthetic urethane derivative of pladienolide D with activity against tumour cell lines and human tumour xenografts (Iwata et al, 2004). Treatment of a drug screen panel of 39 cancer cell lines with pladienolides resulted in profound growth inhibition with greatest activity against breast and lung cancer cell lines. Comparison of the pladienolide sensitivity profile to that of other anticancer agents revealed that pladienolide has a unique and distinct antitumour spectrum. Additionally, a panel of six cancer cell lines made resistant against classic cytotoxic compounds such as camptothecin, etoposide, vincristine, 5-fluorouracil, doxorubicin and cisplatin were just as sensitive to pladienolide as their drug-sensitive parental cell lines. Although in most cell lines, the exact mechanism of drug resistance was not known, at least one cell line overexpresses P-glycoprotein (MDR1 and ABCB1) implying that pladienolides are not a substrate for this ubiquitous drug transporter (Mizui et al, 2004). Administration of variable doses of $2.5-10 \mathrm{mg} \mathrm{kg}^{-1} \mathrm{day}^{-1}$ of pladienolide B for 5 consecutive days in six human tumour xenograft models, representing breast, ovarian, colon and prostate cancer, caused dose-dependent growth inhibition. Moreover, in the breast cancer BSY-1 xenograft, a clear tumour regression with no sign of recurrence for 36 days was noted (Iwata et al, 2004; Mizui et al, 2004; Kotake et al, 2007). The most prominent adverse effects were a reversible weight loss and dose-limiting haematopoietic toxicity.

By using differently tagged pladienolide derivatives as chemical probes, it was discovered that pladienolide interacts with SAP130 of the SF3b subcomplex. However, a partial interaction with additional components of SF3b (SAP145 and SAP155) cannot be ruled out (Kotake et al, 2007). Although the precise functions of SAP130, SAP145 and SAP155 within the U2 snRNP are not clearly defined, it is likely that the activity of SF3b and hence the U2 snRNP is modulated or impaired by pladienolide binding as indicated by the observed time and dose-dependent disturbance of in vivo splicing and the associated growth inhibition. Pladienolide treatment causes an accumulation of unspliced or incompletely spliced pre-mRNAs and gives rise to fewer and larger nuclear speckles and intranuclear sites where splice factors are stored. Similar phenotypes were observed when other spliceosome components like the U1 and U6 snRNAs were knocked down by antisense oligonucleotides (Kotake et al, 2007), or when the SF3b components such as SAP130, SAP145 and SAP155 were downregulated by siRNAs (Kaida et al, 2007).

FR901464 and its methylated derivative spliceostatin A also interact with SF3b, most likely by binding SAP130 or SAP155 (Kaida et al, 2007). Similar to the effects induced by pladienolide, splicing is partially inhibited causing pre-mRNA to accumulate. Whereas upon spliceostatin A treatment, most pre-mRNA species are retained in the nucleus, a subset of unspliced or partially spliced pre-mRNA is prematurely transported to the cytoplasm where it is translated into aberrant proteins. These observations strongly suggest that SF3b is not only involved in splicing but also in the retention of pre-mRNA. FR901464 and most likely spliceostatin A are potent anticancer agents affecting the proliferation of cancer cell lines (Kaida et al, 2007 and references therein), and inhibiting tumour growth in various xenograft models (Nakajima et al, 1996). The observation that cells exposed to FR901464, spliceostatin A and pladienolide showed a cell cycle arrest in G1 and G2/M phases and prompted a study of the involvement of cell cycle regulators, including cyclins, cyclindependent kinases (CDKs) and some positive and negative regulators of these proteins. It was found that cyclin A and CDK levels were unchanged, whereas p16 and p27, both negative regulators of CDKs levels, were increased. In addition, a Cterminally truncated p27 is expressed due to the translation of an aberrantly spliced p27 pre-mRNA. The truncated p27, however, is still capable of inhibiting CDK2 and may be partly responsible for the observed cell cycle arrest, and perhaps the antitumour activity (Kaida et al, 2007).

Clearly, binding of pladienolide and spliceostatin A to SF3b components interferes with the splicing process as well as surveillance mechanisms that are operational in the cell to prevent the synthesis of truncated proteins. On the basis of the promising preclinical data with respect to the potential anticancer activity of pladienolides B and D and E-7107 phase I dose-escalation trials with E-7107 are currently ongoing in Europe and the United States. Apart from determining the classic toxicity end points, extensive 
pharmacodynamic analyses are performed that may help to define appropriate biomarkers to assess the efficacy of these novel agents. The detailed analysis of drug-induced changes in gene expression patterns may give a deeper insight into the mode of action of these drugs and better define the beneficial effects for cancer patients.

\section{CONCLUSIONS}

RNA splicing is an essential and complex cellular process, which is regulated and mediated by many different factors. Now that our understanding of the physiology of the spliceosome increases as

\section{REFERENCES}

Allemand E, Hastings ML, Murray MV, Myers MP, Krainer AR (2007) Alternative splicing regulation by interaction of phosphatase PP2C $\gamma$ with nucleic acid-binding protein YB-1. Nature Struct Mol Biol 14: 630-638

Barbier J, Dutertre M, Bittencourt D, Sanchez G, Gratadou L, de la Grange P, Auboeuf D (2007) Regulation of H-ras splice variant expression by cross talk between the p53 and nonsense-mediated mRNA decay pathways. Mol Cell Biology 27: 7315-7533

Ben-Dov C, Hartmann B, Lundgren J, Valcárcel J (2008) Genome-wide analysis of alternative pre-mRNA splicing. Biol Chem 18: 1229-1233

Blencowe BJ (2006) Alternative splicing: new insights form global analyses. Cell 126: $37-47$

Disher K, Skandalis A (2007) Evidence of the modulation of mRNA splicing fidelity in humans by oxidative stress and p53. Genome 50: $946-953$

Heider KH, Kuthan H, Stehle G, Munzert G (2004) CD44v6: a target for antibody-based cancer therapy. Cancer Immunol Immunother 53: 567 - 579

House AE, Lynch K (2008) Regulation of alternative splicing: more than just the ABCs. J Biol Chemistry 283: 1217-1221

Iwata M, Ozawa I, Uenaka T, Shimizu H, Niijima J, Kanada R, Fukuda Y, Nagai M, Kotake Y, Yoshida M, Tsuchida T, Mizui Y, Yoshimatsu K, Asada M. (2004) E7107, a new urethane derivative of pladienolide D, displays curative effect against several human tumour xenografts. Proc Amer Assoc Cancer Res 45: 691 (abstract 2989)

Jurica MS, Moore MJ (2003) Pre-mRNA splicing: awash in a sea of proteins. Mol Cell 28: $336-345$

Kaida D, Motoyoshi H, Tashiro E, Nojima T, Hagiwara M, Ishigami K, Watanabe H, Kitahara T, Yoshida T, Nakajima H, Tani T, Horinouchi S, Yoshida M (2007) Spliceostatin A targets SF3b and inhibits both splicing and nuclear retention of pre-mRNA. Nature Chem Biol 3: 576-583

Kotake Y, Sagane K, Owa T, Mimori-Kiyosue Y, Shimizu H, Uesugi M, Ishihama Y, Iwata M, Mizui Y (2007) Splicing factor SF3b as a target of the antitumor natural product pladienolide. Nature Chem Biol 3: $570-575$

Laverman P, Roosenburg S, Gotthardt M, Park J, Oyen WJG, de Jong M, Hellmich MR, Rutjes FPJT, van Delft FL, Boerman OC (2008) Targeting of a CCK2 receptor splice variant with 111In-labelled cholecystokinin-8 (CCK8) and 111In-labelled minigastrin. Eur J Med Mol Imaging 35: 386-392

Mizui Y, Sakai T, Iwata M, Uenaka T, Okamoto K, Shimizu H, Yamori T, Yoshimatsu K, Asada M (2004) Pladienolides, new substances from culture of Streptococcus platensis Mer-11107, III. In vitro and in vivo antitumor activities. J Antibiotics (Tokyo) 57: 188-196

Muraki M, Ohkawara B, Hosoya T, Onogi H, Koizumi J, Sumi K, Yomoda J, Murray MV, Kimura H, Furuichi K, Shibuya H, Krainer AR, Suzuki M, Hagiwara M (2004) Manipulation of alternative splicing by a newly developed inhibitor of Clks. J Biol Chem 23: 24246-24254

Nakajima H, Hori Y, Terano H, Okuhara M, Manda T, Matsumoto S, Shimomura K (1996) New antitumor substances, FR901463, FR901464 and FR901465. II. Activities against experimental tumors in mice and mechanism of action. J Antibiot 12: 1204-1211 well as our perception increases how (alternative) splicing may contribute to cancer, we hopefully can find ways to control and intervene with splicing, hereby preventing the expression of carcinogenic proteins. The pladienolide compounds and spliceostatin A that target the spliceosome may very well become the first representatives of a totally new class of anticancer agents.

\section{ACKNOWLEDGEMENTS}

We thank Hans Kneevel and Antonius WM Boersma for their expert help in preparing the figures.
Orban TI, Olah E (2003) Emerging roles of BRCA1 alternative splicing. Mol Pathol 56: $191-197$

Pilch B, Allemand E, Facompré M, Bailly C, Riou JF, Soret J, Tazi J (2001) Specific inhibition of serine- and arginine-rich splicing factors phosphorylation, spliceosome assembly, and splicing by the antitumor drug NB-506. Cancer Res 18: 6876-6884

Rino J, Carvalho T, Braga J, Desterro JMP, Lührmann R, Carmo-Fonseca M (2007) A stochastic view of spliceosome assembly and recycling in the nucleus. PLoS Comput Biol 3: 2019-2031

Singh RN (2007) Evolving concepts on human SMN pre-mRNA splicing. RNA Biology 4: 7-10

Skotheim RI, Nees M (2007) Alternative splicing in cancer: noise, functional or systematic? Int J Biochem Cell Biology 30: 292-302

Soret J, Bakkour N, Maire S, Durand S, Zekri L, Gabut M, Fic W, Divita G, Rivalle C, Dauzonne D, Nguyen CH, Jeanteur P, Tazi J (2005) Selective modification of alternative splicing bt indolederivatives that target the serine-arginine-rich protein splicing factors. Proc Natl Acad Sci USA 102: $8764-8769$

Staley JP, Guthrie C (1998) Mechanical devices of the spliceosome: motors, clocks springs and things. Cell 92: 315-326

Tazi J, Bakkour N, Soret J, Zekri L, Hazra B, Laine W, Baldeyrou B, Lansiaux A, Bailly C (2005a) Selective inhibition of topoisomerase I and various steps of spliceosome assembly by diosporin derivatives. Mol Pharmacol 67: 1186 - 1194

Tazi J, Durand S, Jeanteur P (2005b) The spliceosome: a novel multi-faceted target for therapy. Trends Biochem Sci 30: 469-575

van Deutekom JC, Janson AA, Ginjaar IB, Frankhuizen WS, Aartsma-Rus A, Bremmer-Bout $\mathrm{M}$, den Dunnen JT, Koop K, van der Kooi AJ, Goemans NM, de Kimpe SJ, Ekhart PF, Venneker EH, Platenburg GL, Verschuuren JJ, van Ommen GJ (2007) Local dystrophin restoration with antisense oligonucleotide PRO051. $N$ Engl $J$ Med 357: $2677-2686$

Vela E, Hilari JM, Roca X, Muñoz-Mármol AM, Ariza A, Isamat M (2007) Multisite and bidirectional exonic splicing enhancer in CD44 alternative exon v3. RNA 13: 1-12

Wang Z, Burge CB (2008) Splicing regulation: from a parts list or regulatory elements to an integrated splicing code. RNA 14: $802-813$

Will CL, Lührmann R (2001) Spliceosomal UsnRNP biogenesis, structure and function. Currt Opin in Struct Biol 13: 290-301

Xiang C, Sarid R, Cazacu S, Finnis S, Lee H-K, Ziv-Av A, Mikkelsen T, Brodie C (2007) Cloning and characterization of human RTVP-1b, novel splice variant of RTVP-1 in glioma cells. Biochem and Biophys Res Commun 362: 612-618

Yu C-P, Yu J-C, Sun C-A, Tzao C, Ho J-Y, Yen AM-F (2007) Tumor susceptibility and prognosis of breast cancer associated with the G870A polymorphism of CCND1. Breast Cancer Res Treat 107: 95-102

Zhou Z, Licklider LJ, Gygi SP, Reed R (2002) Comprehensive proteomic analysis of the human spliceosome. Nature 419: 182-185 